\title{
Ação Crônica do Ácido Acetilsalicilico na Prenhez da Rata
}

\author{
Chronic Effects of Acetylsacylic Acid on Pregnant Rats
}

\begin{abstract}
Sílvia Espiridião ${ }^{1}$, Renato Ajeje ${ }^{2}$, Benjamin I. Kopelman ${ }^{3}$, Manuel J. Simões ${ }^{2}$,
\end{abstract} Joaquim Evêncio-Neto ${ }^{2}$, Luiz Kulay Júnior ${ }^{2}$

\begin{abstract}
RESUM0
O objetivo deste trabalho foi avaliar os efeitos do ácido acetilsalicílico (AAS) sobre a prenhez da rata albina. Para tanto, utilizamos 60 ratas prenhes divididas ao acaso em seis grupos numericamente iguais. Todas receberam diariamente por gavagem o volume total de $1 \mathrm{ml}$, do $5^{\circ}$ até o $20^{\circ}$ dia de prenhez, com as seguintes características: Grupo I - somente água destilada (controle); Grupo II - solução aquosa de carboximetilcelulose 0,2\% (veículo); Grupos III, IV, V e VI - respectivamente, $1,10,100$ e $400 \mathrm{mg} / \mathrm{kg}$ de peso de AAS dissolvido em solução de carboximetilcelulose a $0,2 \%$. Os pesos maternos foram anotados nos dias zero, $7^{\circ}, 14^{\circ}$ e $20^{\circ}$ de prenhez. No $20^{\circ}$ dia as matrizes foram sacrificadas. Nossos resultados mostraram diminuição do número de crias vivas a partir da administração de $100 \mathrm{mg} / \mathrm{kg}$ de AAS e também queda do ganho de peso materno, dos conceptos e placentas nas ratas tratadas com dose de $400 \mathrm{mg} / \mathrm{kg}$ por dia.
\end{abstract}

PALAVRAS-CHAVE: Ácido acetilsalicílico. Droga na gestação. Analgésicos. Placenta.

\section{Introdução}

Agente analgésico, antipirético e antiinflamatório, o ácido acetilsalicílico (AAS) tem consumo estimado da ordem de 40 mil toneladas anuais. Considerando o universo de 54 mil gestantes, pode-se dizer que $60 \%$ consumiram o

\footnotetext{
${ }^{1}$ Departamento de Saúde Materno-Infantil da Faculdade de Medicina do ABC, São Paulo.

2 Departamento de Tocoginecologia da Universidade Federal de São Paulo/Escola Paulista de Medicina, São Paulo.

${ }^{3}$ Departamento de Pediatria da Universidade Federal de São Paulo/Escola Paulista de Medicina, São Paulo.

Correspondência:

Luiz Kulay Júnior

Rua Napoleão de Barros, $715,8^{\circ}$ andar.

Hospital São Paulo, Disciplina de Obstetrícia.

CEP 04024-002, São Paulo-SP, Brasil.
}

produto em algum período da gravide $z^{12}$.

O AAS ultrapassa a placenta com facilidade ${ }^{18}$. Trabalhos clínicos, no entanto, têm relatado efeitos contraditórios com relação ao binômio maternofetal. No organismo materno são descritos efeitos adversos, como aumento da incidência de gravidez prolongada, prolongamento do trabalho de parto ${ }^{6}$ e hemorragias pós-parto ${ }^{7}$. Com relação ao concepto os achados são maior freqüência do retardo de crescimento intra-uterino (RCIU), mortalidade perinatal $^{4}$ e hemorragias dos recém-nascidos ${ }^{2,20}$. Por outro lado, em circunstâncias semelhantes, Kullander e Kallen ${ }^{15}$ nada relataram.

Quanto aos trabalhos experimentais, doses variadas administradas em diversos períodos da prenhez da rata sempre revelaram efeitos adversos, como aumento do índice de malformações 
esqueléticas e do sistema nervoso central, de reabsorções e da mortalidade do concepto, a par da diminuição do peso das placentas e das crias $^{1,3,9,25}$.

O objetivo deste trabalho é o de avaliar os efeitos sobre a matriz e respectivos conceptos sob doses variáveis de AAS ministradas durante toda a prenhez da rata.

\section{Material e métodos}

\section{Animais e tratamento}

Utilizamos ratas (EPM-1 Wistar), adultas, virgens, pesando de 150 a $200 \mathrm{~g}$, com regime de alimentação (ração Purina-Labina) e de água à vontade. Foram inicialmente acasaladas na proporção de duas fêmeas para cada macho por um período de duas horas. O teste de prenhez foi determinado pelo encontro de espermatozóides na luz vaginal. Sessenta ratas foram então divididas ao acaso em seis grupos numericamente iguais, a saber: GI, animais que receberam água (controle); GII, animais que receberam solução aquosa de carboximetilcelulose (CMC) a 0,2\% (veículo do AAS); GIII, animais tratados com $1 \mathrm{mg} / \mathrm{kg}$ de AAS (sal cedido pela BAYER do Brasil, lote $\mathrm{n}^{\circ}$ 910191, teor de pureza de 99,5\%); GIV, animais tratados com $10 \mathrm{mg} / \mathrm{kg}$ de AAS; GV, animais tratados com 100 $\mathrm{mg} / \mathrm{kg}$ de AAS e GVI, animais tratados com 400 $\mathrm{mg} / \mathrm{kg}$ de AAS. Em todos os grupos o AAS foi dissolvido em $\mathrm{CMC}$ a $0,2 \%$. O tratamento teve início no quinto dia após a positividade do teste de prenhez ( 0 dia) e se estendeu até o $20^{\circ}$ dia, sendo que cada um dos animais de qualquer dos grupos estudados recebeu diariamente sempre o volume total de $1 \mathrm{ml}$ de solução, por gavagem, independente do grupo apreciado.

Todas as ratas foram pesadas nos dias 0,7 , 14 e 20 de prenhez. O ganho de peso percentual foi calculado de acordo com a seguinte fórmula:

\section{D\% = (peso no dia $\underline{X}$ - peso inicial) $\times 100 /$ peso inicial onde $\underline{X}=7^{\circ}$ ou $14^{\circ}$ ou $20^{\circ}$ dia de prenhez.}

Ao termo $\left(20^{\circ} \mathrm{dia}\right)$, todos os animais foram sacrificados sob anestesia profunda com éter etílico; após laparotomia seguida de histerectomia, foram anotados os seguintes parâmetros: número de sítios de implantação e de reabsorção, número de placentas e de fetos e determinados os pesos das placentas e dos fetos.

\section{Análise estatística}

Análises paramétricas e não-paramétricas foram realizadas; o nivel de rejeição para hipótese de nulidade foi fixada em 5\%. Múltiplas comparações foram feitas pelo teste de KruskalWallis e análise de variância.

\section{Resultados}

Os ganhos de peso das ratas foram significantemente menores nos animais que receberam doses superiores a $100 \mathrm{mg}$ de AAS $/ \mathrm{kg}$ de peso corporal por dia e podem ser vistos na Figura 1.

Quanto ao número de fetos e seus pesos individuais médios houve diminuição quando expostos a 100 e $400 \mathrm{mg} / \mathrm{kg}$ de peso por dia. O peso individual médio das placentas apresentou diminuição somente no grupo VI, que recebeu 400 $\mathrm{mg}$ de AAS $/ \mathrm{kg}$ de peso durante todo o curso da prenhez (Tabela 1).

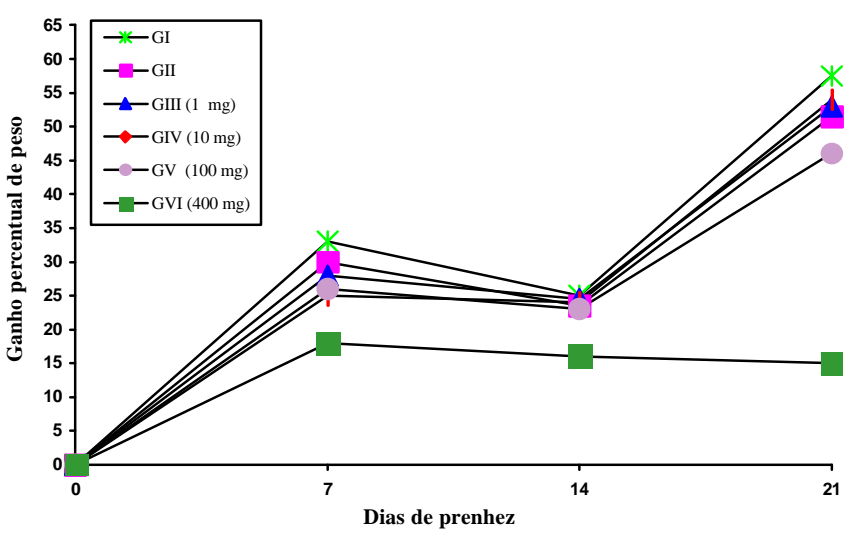

Figura 1 - Ganho de peso $(\mathrm{g})$ das ratas prenhes tratadas com as várias doses de ácido acetilsalicílico. (GVI $\neq$ dos outros, $p<0,05)$

\section{Discussão}

A escolha, em nosso trabalho, das doses de 1,10 e $100 \mathrm{mg} / \mathrm{kg}$ de peso corporal por dia está relacionada às várias indicações clínicas, isto é: $1 \mathrm{mg} / \mathrm{kg}$ por dia, utilizada atualmente na prevenção da moléstia hipertensiva específica da gravide $z^{22,27}$; $10 \mathrm{mg} / \mathrm{kg}$ por dia, usada como analgésico e antipirético ${ }^{29} ; 100 \mathrm{mg} / \mathrm{kg}$ por dia é empregada como antiinflamatório, ao passo que $400 \mathrm{mg} / \mathrm{kg}$ dia é considerada dose tóxica ${ }^{21}$. 
Tabela 1 - Efeitos do ácido acetilsalićlico sobre os vários parâmetros estudados na rata prenhe.

GI

$96,17 \pm 3,22$

$100,00 \pm 0,00$

$93,33 \pm 4,44$

$98,75 \pm 1,25$

$03,36 \pm 0,12$

$03,59 \pm 0,08$

$03,33 \pm 0,08 *$

$01,90 \pm 0,14 * *$

fetos $(\mathrm{g})$

Peso individual médio das

$00,47 \pm 0,01$

$000,42 \pm 0,02$

$00,45 \pm 0,01$

$00,46 \pm 0,02$

$00,46 \pm 0,01$

$00,35 \pm 0,03^{*}$

placentas $(\mathrm{g})$

* Significantemente diferente dos outros grupos $(\mathrm{p}<0,05)$

** Significantemente diferente dos outros grupos $(\mathrm{p}<0,05)$
Os salicilatos ultrapassam com facilidade a membrana sinciciocapilar, conforme demonstrado tanto pelo uso da aspirina ${ }^{14} \mathrm{C}$ quanto por sua avaliação tecidual no concepto ${ }^{14,24}$.

Wolf et al. ${ }^{28}$ avaliaram que o nível de AAS no plasma fetal é aproximadamente um terço do nível encontrado no plasma materno e que o nivel do seu principal metabólito, ainda mais potente, o ácido salicílico, corresponde a 80 - 90\% do nível materno. Tal fato poderia justificar a toxicidade da droga no feto, pela rápida desesterificação hepática transformando o AAS em ácido salicílico. De Sweit e Fryers ${ }^{8}$ referiram ainda que $50 \%$ do AAS se transforma em ácido salicílico a cada 20 minutos no organismo materno, aumentando, como conseqüência, o teor no plasma fetal.

Deve ser mencionado que o rato é um animal muito tolerante ao AAS, uma vez que a DL 50 é de $1,75 \mathrm{~g} / \mathrm{kg}$ de peso ${ }^{5}$. Morikawa et al. ${ }^{17}$, por sua vez, referem que a dose minima que atua no rato como antiagregante plaquetária é de $10 \mathrm{mg} / \mathrm{kg}$ em machos e de $20 \mathrm{mg} / \mathrm{kg}$ em fêmeas. Neste experimento, a droga foi administrada do $5^{\circ}$ ao $20^{\circ}$ dia de prenhez, período que engloba as fases de embriogênese e desenvolvimento do concepto.

No que se refere à administração de 1 e 10 $\mathrm{mg}$ de AAS $/ \mathrm{kg}$ de peso corporal por dia não foram observados efeitos adversos sobre a prenhez da rata, consideradas as variáveis ganho de pesos materno, do concepto, da placenta, assim como, taxa de vitalidade das crias. No entanto, com as doses de 100 e de $400 \mathrm{mg} / \mathrm{kg}$ obtivemos número menor de fetos associado a baixo peso, quando comparados aos demais grupos estudados (Tabela 1). Assim, em nosso estudo foi considerada baixa a dose diária $1 \mathrm{mg} / \mathrm{kg}$, dose moderada $10 \mathrm{mg} / \mathrm{kg}$ e doses elevadas a partir de $100 \mathrm{mg} / \mathrm{kg}$.

Collins e Turner ${ }^{6}$ referiram que os recémnascidos de mulheres que ingeriram salicilatos durante a gravidez apresentaram aumento significante das taxas de natimortos e de mortalidade perinatal. O efeito de doses elevadas de salicilatos é amplamente discutido na literatura; em humanos, pode ocorrer toxicidade com ingestão que varia desde 500 até $2000 \mathrm{mg} / \mathrm{kg}$ de $A A S^{11}$. Deve ser mencionado que esses dados apoiam os nossos achados no que diz respeito ao número e peso dos fetos, quando utilizamos as altas doses do AAS, relembrando ainda que, em ratas, no caso de óbito intra-uterino, pode haver reabsorção total dos conceptos em qualquer período da gravidez.

Na literatura consultada, não encontramos trabalhos experimentais mostrando qualquer tipo de ação tóxica do AAS na placenta. Pelo contrário, é interessante relatar que Wallenburg e Rotmans ${ }^{27}$, administrando baixas doses de AAS em humanos, preveniram a insuficiência útero-placentária idiopática. Quanto ao peso individual médio das placentas, obtivemos queda estatisticamente significante somente entre os animais do grupo que recebeu $400 \mathrm{mg} / \mathrm{kg}$; esse achado, ao que parece, deve estar associado ao grau de toxicidade do fármaco.

Quando se trata, porém, de ácido acetilsalicílico é impositivo considerar o papel das prostaglandinas, compostos derivados do ácido araquidônico metabolizado no fígado pelas enzimas ciclooxigenase (COX). As prostaglandinas $\mathrm{PGD}_{2} \mathrm{e}$ $\mathrm{PGI}_{2}$ são sintetizadas respectivamente com maior intensidade no endotélio vascular e mastócitos da decídua materna, membranas fetais e cordão umbilical $^{26}$, e são responsáveis pela vasodilatação, 
relaxamento do útero e inibição da agregação plaquetária.

As ciclooxigenases, por sua vez, existem sob duas isoformas: ciclooxigenase $1\left(\mathrm{COX}_{1}\right)$ enzima presente na grande maioria das células, está envolvida na homeostase normal, regulando as respostas circulantes principalmente dos glicolíticos, glucagon, adrenalina e vasopressina, promovendo a elevação da glicemia $^{10}$; ciclooxigenase $2\left(\mathrm{COX}_{2}\right)$ estimulada pelas células dos processos inflamatórios raramente é encontrada em processos fisiológicos ${ }^{11}$.

O AAS em doses altas assim chamadas, antiinflamatórias, inibe por sua vez, de forma irreversivel, a $\mathrm{COX}_{1}$, por acetilação da serina em posição $530^{16}$, o que implica na liberação de substrato, via lipoxigenase, que atua sobre plaquetas, músculos lisos e células endoteliais, alterando a homeostase ${ }^{13}$; além disso, pode haver também efeito vasoconstritor secundário, por inibição da conversão do ácido 12-L-hidroperoxi $5,8,10,14$ eicosatetróico (12-HPETE) para ácido 12-L-hidroxi 5, 8, 10, 14 eicosatetróico (12-HETE), conforme Seigel et al. ${ }^{23}$. Além disso, o AAS atua sobre os processos de fosforilação oxidativa aumentando o consumo de $\mathrm{O}_{2}$, como também interfere no equilíbrio ácido-básico e de eletrolitos ${ }^{19}$.

Finalizando, queremos dizer que, esta sucessão de fatos adversos por nós encontrada no binômio materno-fetal como a diminuição tanto dos pesos das ratas quanto dos baixos pesos das crias e das placentas, como também, da redução do número de crias vivas, podem ser creditados aos distúrbios do mecanismo homeostático por ingestão de altas doses de AAS durante toda a prenhez.

\section{SUMMARY}

The purpose of the present study was to evaluate the effects of acetylsalicylic acid (ASA) on the pregnancy of female albino rats. We used 60 pregnant female rats which were divided into six groups of ten cache. All the animals received daily by gavage, from the $5^{\text {th }}$ (day zero) until the $20^{\text {th }}$ day of pregnancy, $1 \mathrm{ml}$ of the following: Group I - only distilled water (control); Group II - $0.2 \%$ aqueous solution of carboxymethylcellulose (vehicle); Groups III, IV, V and VI - 1, 10, 100 and $400 \mathrm{mg} / \mathrm{kg}$ body weight respectively, of ASA diluted in $0.2 \%$ carboxymethylcellulose solution. The animals were weighed on days 0, 7, 14 and 20 of pregnancy. Our results showed that the animals treated with $100 \mathrm{mg}$ of ASA presented a reduction in the number of live newborns. The animals treated with 400 $\mathrm{mg} / \mathrm{kg} /$ day presented not only a reduction in the number of live newborns but also decrease in maternal, newborn and placental weight.

KEY WORDS: Acetylsalicylic acid. Placenta. Analgesic. Drugs in pregnancy.

\section{Referências}

1.Baba T, Nagahama M, Akiyama N, Miki T. Experimental production of malformations due to acetyl salicylate and phenyl salicylate in rats. Osaka City Med J 1966; 12:23-9.

2.Bleyer WA, Breckenridge RT. Studies on the detection of adverse drug reactions in the newborn. II. The effects of prenatal aspirin on newborn hemostasis. JAMA 1970; 213:2049-53.

3.Brown RA, West GB Effect of acetylsalicylic acid on foetal rats. J Pharmacol 1964; 16:563-5.

4.Cekanova E, Larsson KS, Morck E, Aberg G. Interactions between salicylic acid and pyridyl-3-methanol: antinflammatory and teratogenic effects. Acta Pharmacol Toxicol 1974; 35:107-18.

5.Circurel L, Schmid B. In vitro teratogenicity of acetylsalicylic acid on rat embryos: studies with various culture conditions. Methods Find Exp Clin Pharmacol 1986; 8:227-32.

6.Collins E, Turner G. Maternal effects of regular salicylate ingestion in pregnancy. Lancet 1975; 2:335-7.

7.Corby DG. Aspirin in pregnancy: maternal and fetal effects. Pediatrics 1978; 62:930-7.

8.De Swiet M, Fryers G. Review: The use of aspirin in pregnancy. J Obstet Gynecol 1990; 10:457-82.

9.Ericksson M. Salicylate-induced foetal damage late in pregnancy. An experimental study in mice. Acta Paediat Scand 1971 Suppl; 211:8-24.

10.Gomez-Foix AM, Rodriguez-Gil JE, Guinovart JJ, Bosch F. Prostaglandins E2 and F2 alpha affect glycogen synthase and phosphorylase in isolated hepatocytes. Biochem J 1989; 261:93-7.

11.Goodman LS, Gilman A. The pharmacological basis of therapeutics. 9 ${ }^{\text {nd }}$ ed. New York: Mc Graw-Hill; 1996. p. 601-30.

12.Harrison K, Thomas I, Smith I. Analgesic use during pregnancy. Med J Aust 1978; 2:161.

13.Klein KL, Clark KE, Scott Jr WJ. Prostaglandin synthesis in rat embryo tissue: the effect of non-steroidal anti-inflammatory drugs in vivo and ex vivo. Prostaglandins 1984; 27:659-72.

14.Koshakjy RP, Schulert AR. Biochemical mechanisms of salicylate teratology in the rat. Biochem Pharmacol 1973; 22:407-16.

15.Kullander S, Kallen B. A prospective study of drugs and pregnancy. 4. Miscellaneous drugs. Acta Obstet Gynecol Scand 1976; 55:287-95.

16. Meade EA, Smith WL, De Witt DL. Differential inhibition of prostaglandin endoperoxide synthase (ciclooxygenase) isoenzymes by aspirin and other non-steroidal anti-inflammatory drugs. J Biol Chem 1993; 268:6610-4. 
17.Morikawa M, Kojima T, Inoue M, Tsuboi M. Comparison of the inhibitory effects of aspirin and triclopidine on platelet aggregation and $\mathrm{Ca} 2+$ mobilization in rat platelets. Res Commun Chem Pathol Pharmacol 1986; 54:275-8.

18.Palmisano PA, Cassady G. Salicylate exposure in the perinate. JAMA 1969; 209:556-8.

19.Rang HP, Dale MM, Ritter JM. Pharmacology. $3^{\text {rd }}$ ed. Edimburgh: Churchill-Livingstone; 1995. p. 253-4.

20.Rumack CM, Guggenheim MA, Rumack BH, Peterson RG, Johnson ML, Braithwaite WR Neonatal intracranial hemorrhage and maternal use of aspirin. Obstet Gynecol 1981; 58:52S-6S.

21.Schaller JG. Chronic salicylate administration in juvenile rheumatoid arthritis: aspirin hepatitis and its clinical significance. Pediatrics 1978; 62:916-25.

22.Sibai BM. An aspirin a day to prevent prematury. Clin Perinatol 1992; 19:305-17.

23.Siegel MI, Mc Connell RT, Porter NA, Cuatrecasas P. Arachidonate metabolism via lipoxygenase and 12 L-hydroperoxy-5,8,10,14-icosatetraenoic acid peroxidase sensitive to anti-inflammatory drugs. Proc Natl Acad Sci USA 1980; 77:308-12.
24.Tagashira E, Nakao K, Urano T, Ishikawa S, Hiramori T, Yanaura S. Correlation of teratogenicity of aspirin to the stage-specific distribution of salicylic acid in rats. Jpn J Pharmacol 1981; 31:563-71.

25.Takacs E, Warkany J. Experimental production of congenital malformations in rats by salicylate poisoning. Teratology 1968; 1:109-18.

26.Vane J, Botting R. Inflammation and the mechanism of action of anti-inflammatory drugs. FASEB $\mathbf{J}$ 1987; 1:89-96.

27.Wallenburg HC, Rotmans N. Prevention of recurrent idiopathic fetal growth retardation by low-dose aspirin and dipyridamole. Am J Obstet Gynecol 1987; 157:1230-5.

28.Wolf F, Berg R, Putter J. Pharmacokinetics studies on the placental transfer of acetylsalicylic acid intravenously administered in the prepartal period. Arch Gynecol 1977; 224:195-6.

29.Woodbury DW. Analgésicos, antipiréticos, agentes antiinflamatórios e inibidiores da síntese do ácido úrico. In: Goodman LS, Gilman A. As bases da farmacologia terapêutica. $4^{\text {th }}$ ed. Rio de Janeiro: Guanabara Koogan; 1973. p. 308. 